\title{
Enhancement of stratospheric aerosols after solar proton event
}

\author{
O. I. Shumilov ${ }^{1}$, E. A. Kasatkina ${ }^{1}$, K. Henriksen ${ }^{2, *}$, E. V. Vashenyuk ${ }^{3}$ \\ ${ }^{1}$ High-Latitude Geophysical Laboratory of St. Petersburg Filial of IZMIRAN, Post Box 123, 184200 Apatity, Russia \\ 2 The Auroral Observatory, University of Tromso, 9037 Tromso, Norway \\ ${ }^{3}$ Polar Geophysical Institute, Apatity, Russia
}

Received: 23 August 1995/Revised: 27 March 1996/Accepted: 17 April 1996

\begin{abstract}
The lidar measurements at Verhnetulomski observatory $\left(68.6^{\circ} \mathrm{N}, 31.8^{\circ} \mathrm{E}\right)$ at Kola peninsula detected a considerable increase of stratospheric aerosol concentration after the solar proton event of GLE (ground level event) type on the $16 / 02 / 84$. This increase was located at precisely the same altitude range where the energetic solar protons lost their energy in the atmosphere. The aerosol layer formed precipitated quickly $(1-2 \mathrm{~km}$ per day) during 18,19 , and 20 February 1984, and the increase of $R(H)$ (backscattering ratio) at $17 \mathrm{~km}$ altitude reached $40 \%$ on $20 / 02 / 84$. We present the model calculation of $C N$ (condensation nuclei) altitude distribution on the basis of an ion-nucleation mechanism, taking into account the experimental energy distribution of incident solar protons. The meteorological situation during the event was also investigated.
\end{abstract}

\section{Introduction}

It is now well documented that heterogeneous chemistry triggering ozone destruction occurs on sulfate aerosols (Tolbert et al., 1988; Hofman and Solomon, 1989). The main sources of sulfate aerosols in atmosphere are volcanic eruptions, anthropogenic emissions and biological activity. Contribution of each component to aerosol layer formation in the stratosphere differs in the two hemispheres and depends on latitude. The anthropogenic pollution seems to be the major contributor of sulfuric acid in Arctic region compared with Antarctic where the phytoplankton emissions seem to prevail (Langner et al., 1992). However, volcanos also sometimes play a dominant role as sources of sulfur injections into the stratosphere. For example, the eruption of Mount Pinatubo (1991) caused increases in sulfuric acid abundance by a factor of 50 (Tolbert, 1994). Another source of stratospheric aerosols

Correspondence to: E. A. Kasatkina

*deceased exists in nature besides those already mentioned. The mechanism of ion nucleation triggering by solar cosmic ray ionization was suggested by Arnold (1982) as a potential source of condensation nuclei $(C N)$ on which stratospheric sulfate aerosols can form (Arnold, 1982; Hofman and Rosen, 1983).

Ion nucleation involves growth of ions by association of sulfuric acid molecules to a critical size at which the resulting molecular cluster is stable against ion-ion recombination. The rate of formation of nuclei is proportional to the ion production rate, and thus temporal and spatial changes of atmospheric ionization due to solar and galactic cosmic rays may induce corresponding changes of condensation nuclei concentration.

The creation of sulfate aerosols by means of ion nucleation mechanism seems to be a possible reason for the ozone "minihole" appearance resulting from a set of heterogeneous chemical reactions after solar proton events of GLE type in May 1990 at Spitsbergen (Shumilov et al., 1992; 1995). In this work the mechanism of aerosol formation during the GLE event on 16 February 1984 is discussed.

\section{Experimental results}

The solar proton event (SPE) of 16 February 1984 was detected at 08:58 UT and was caused by a solar flare behind the $\mathrm{W}$-limb of the Sun. It had a very rigid energy spectrum and belonged to the GLE type of SPE. The maximum intensity of solar protons during the GLE reached 5 particles $\mathrm{cm}^{-2} \mathrm{~s}^{-1} \mathrm{ster}^{-1}$ for energies between 84 and $200 \mathrm{MeV}$ (Hargreaves et al., 1987). Figure 1 shows the Apatity $\left(\Phi^{\prime}=63.3^{\circ}\right)$ neutron monitor data for February 1984.

During February 1984 the stationary lidar operated at Verhnetulomski observatory $\left(68.6^{\circ} \mathrm{N}, 31.8^{\circ} \mathrm{E}\right)$ located in the Murmansk region. At the lidar the diameters of receiving and transmitting telescopes were $50 \mathrm{~cm}$ and $20 \mathrm{~cm}$, respectively. Two lasers with emission wavelengths of $694.3 \mathrm{~nm}$ and $532.0 \mathrm{~nm}$ were used in the lidar. All profiles 


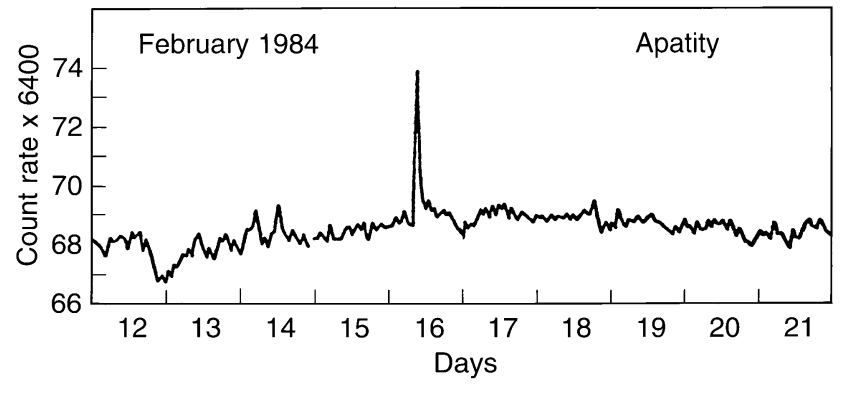

Fig. 1. Hourly count rates of the neutron monitor at Apatity observatory from 12 to 21 February 1984

shown here relate to the $694.3 \mathrm{~nm}$ channel. The ruby laser has wavelength of $694.3 \mathrm{~nm}$, pulse repetition $-1 \mathrm{~Hz}$, energy per pulse $-0.4 \mathrm{~J}$ and pulse width $-30 \mathrm{~ns}$. To limit the background emission an interference filter with $1.8 \mathrm{~nm}$ bandwidth and 0.4 transmission coefficient is used in the receiver. Lidar permits measurements in an altitude range between 3 and $40 \mathrm{~km}$ with vertical resolution of $1.5 \mathrm{~km}$.

In Fig. 2 profiles of aerosol backscatter ratio $R(H)$ at wavelength of $694.3 \mathrm{~nm}$ are shown. The expression for aerosol backscatter ratio is as follows:

$R(H)=\left(\beta_{\text {mol }}+\beta_{\text {aer }}\right) / \beta_{\text {mol }}$,

where $\beta_{m o l}$ and $\beta_{a e r}$ are molecular and aerosol backscattering respectively. Figure 2 shows $15,18,19$, and 20 February $R(H)$ profiles relative to the curve of average values for previous five undisturbed days. The lidar measurements on 16 and 17 February were absent because of bad weather conditions. The aerosol backscatter increase on 18 February 1984 between 15 and $25 \mathrm{~km}$ altitude can be seen (Fig. 2).

To examine the influence of meteorological factors an analysis of the meteorological situation over the Kola peninsula region has been made. The $100-\mathrm{hPa}$ meteorological map analysis has shown that during the period from 13 February to 20 February, the Kola peninsula was in the coldest part of the circumpolar vortex. Some altitude temperature profiles are shown in Fig. 3. These profiles were obtained at Murmansk meteorological observatory $\left(68.3^{\circ} \mathrm{N}, 33^{\circ} \mathrm{E}\right)$ located close to Verhnetulomski observatory. Figure 3 clearly shows that the temperatures are low enough for PSC formation.

Additional information about the meteorological situation before and after GLE event considered may be obtained from Fig. 4 where the $100-\mathrm{hPa}$ (16-km altitude) temperatures for Kola peninsula during the second half of February 1984 are shown. The arrows indicate the temperature at which Type-1 PSCs can be formed (Nagatani et al., 1990). Figure 4 demonstrates that the 100-hPa temperatures were sufficiently low, and PSC activity could be expected over Kola peninsula between 13 February and 21 February. However, the aerosol enhancement seems not to be related to the temperature decrease on 16 February $\left(-84^{\circ} \mathrm{C}\right)$. The same extreme cold temperature was observed on 13 February $\left(-83^{\circ} \mathrm{C}\right)$ without any aerosol backscatter ratio $R(H)$ changes (for reference see Figs. 2,3). It seems reasonable to say that the PSC could form even before 13 February, and the additional temper-
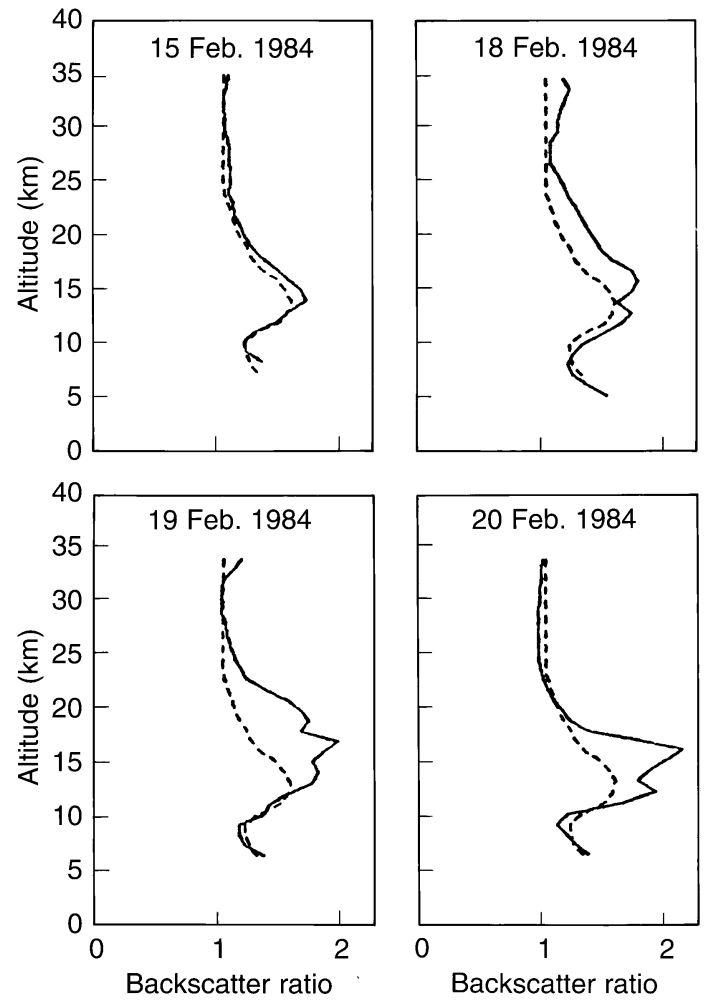

Fig. 2. The vertical profiles of scattering ratio $R(H), l=694.3 \mathrm{~nm}$ taken on 15, 18, 19, and 20 February 1984 at Verhnetulomski observatory. Dashed curves give the quiet day level averaged for previous five undisturbed days
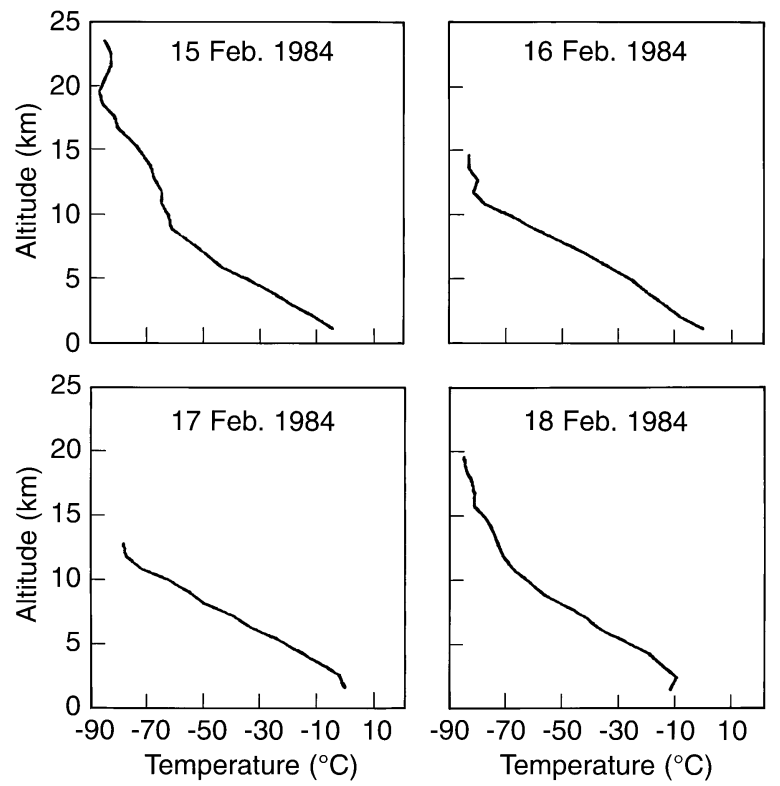

Fig. 3. Temperature profiles measured by balloons on 15, 16, 17, and 18 February 1984 above Murmansk observatory

ature decrease observed on 16 February could not lead to PSC formation. We therefore assume the aerosol increase observed between $15 \mathrm{~km}$ and $20 \mathrm{~km}$ was probably caused by a GLE event. 


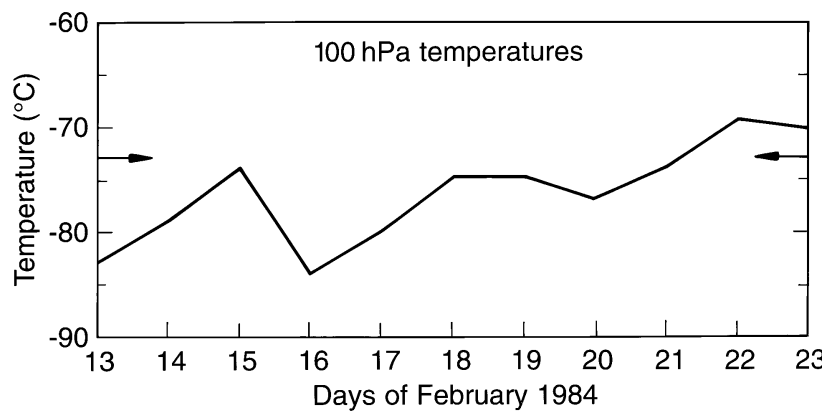

Fig. 4. 100-hPa temperatures over Kola peninsula from 13 February to 23 February 1984 . Arrows indicate the threshold temperatures for type 1 PSC formation

\section{Data analysis and discussion}

Figure 2 shows aerosol backscatter increase on 18 February 1984 relative to the average profile for the previous 5 days. The increase was not associated with considerable temperature enhancements (see Fig. 3) and seems to be caused by the GLE event on 16 February 1984.

Taking into consideration that $\beta_{m o l}=n_{m} \sigma_{m}$ and $\beta_{a e r}=n_{a} \sigma_{a}$, where $\sigma_{m}$ and $\sigma_{a}$ are molecular and aerosol backscattering cross sections, and $n_{m}, n_{a}$ are molecular and aerosol concentrations, respectively, Eq. (1) can be rewritten as:

$R-1=\left(\sigma_{a} n_{a}\right) /\left(\sigma_{m} n_{m}\right)$.

Aerosol concentration can be derived from backscattering ratio at $694.3 \mathrm{~nm}\left(R_{0.69}\right)$. A reasonable aerosol backscattering cross section $\sigma_{a}=2.5 \cdot 10^{-11} \mathrm{~cm}^{2} \mathrm{ster}^{-1}$ and molecular backscattering cross section $\sigma_{m}=$ $2 \cdot 10^{-28} \mathrm{~cm}^{2} \mathrm{ster}^{-1}$ at the $694.3 \mathrm{~nm}$ wavelength are taken from DeLuisi et al. (1975) and Kent and Wright (1970) respectively. Substituting $\sigma_{a}$ and $\sigma_{m}$ and assuming them to be independent of altitude, from Eq. (2) we obtain:

$n_{a}=0.8 \cdot 10^{-17}\left(R_{0.69}-1\right) n_{m}$.

Using the backscatter ratio profile $R_{0.69}(H)$ and a simple model of the atmosphere (Russel et al., 1979), we have calculated from Eq. (3) the height profile of aerosol concentration.

In Fig. 5 the experimental aerosol background profile $(\mathrm{Nb})$ and disturbed aerosol profile $(\mathrm{Nd})$ of 18 February 1984 are shown. These experimental values correspond to ordinary altitude distribution of aerosol particles with sizes $r \geq 0.69 \mu \mathrm{m}$ (Hofman et al., 1983). Calculated aerosol densities have errors limited by 4\% (DeLuisi et al., 1975; Bajdalov, 1978). Figure 5 shows that aerosol concentration increase on 18 February 1984 was about $50 \%$.

To calculate ion production rate $Q\left(\mathrm{~cm}^{-3} \mathrm{~s}^{-1}\right)$ we have used the following equation:

$Q(H)=(2 \pi / \varepsilon) \cdot \int_{E_{1}}^{\infty} D(E) E_{\mathrm{Li}}(E) d E$,

where $E_{\mathrm{Li}}(E)$ is the energy loss in the $i$ th altitude slab in $\mathrm{MeV}$ (Jackman et al., 1980), and $\varepsilon=35 \mathrm{eV}$ is the energy

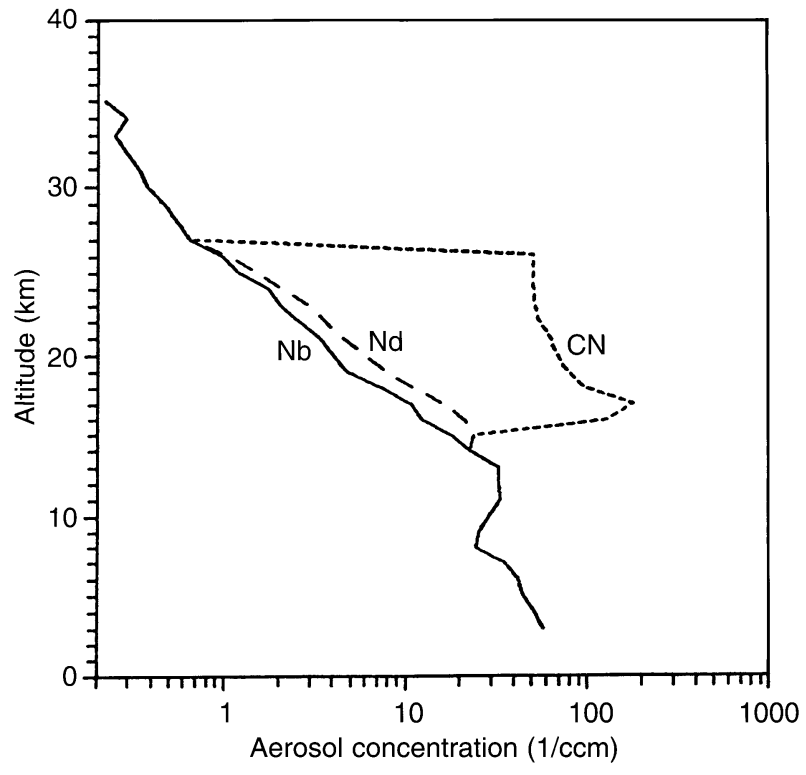

Fig. 5. Aerosol concentration profiles calculated from experimental lidar measurements on 15 February $1984(\mathrm{Nb})$ is quiet day level, and on 18 February $1984(N d)$ is disturbed level. Curve $C N$ (condensation nuclei) gives $C N$ concentration profile calculated for GLE event using ion nucleation mechanism

needed for one ion-electron pair production. $D(E)$ is the differential energy spectrum of incident protons $\left(\mathrm{cm}^{-2} \mathrm{~s}^{-1} \mathrm{sr}^{-1} \mathrm{MeV}^{-1}\right)$ taken from Hargreaves et al. (1987), and $E_{1}$ is the energy of Murmansk observatory geomagnetic cut-off calculated as $150 \mathrm{MeV}$ approximately.

Figure 6 shows the ion production rate profile calculated at the maximum intensity on 16 February 1984 GLE event for $E_{1}=150 \mathrm{MeV}$ (solid curve) and for $E_{1}=0$ (dashed curve). $E_{1}=0$ for geomagnetic polar cap region because the solar protons at all energy levels have free access to an atmosphere there. From Fig. 6 it is clear that these two curves have a considerable difference only above $30 \mathrm{~km}$ altitude.

Ion nucleation rate $J\left(\mathrm{~cm}^{-3} \mathrm{~s}^{-1}\right)$ is related to ion production rate $Q$ by the equation (Hofman and Rosen, 1983):

$J=Q \cdot\left(1+(\alpha Q)^{1 / 2} / K\left[\mathrm{H}_{2} \mathrm{SO}_{4}\right]\right)^{-N c}$,

where $\alpha$ is the ion recombination coefficient $\left(10^{-7} \mathrm{~cm}^{3} \mathrm{~s}^{-1}\right), K$ is the association rate coefficient $\left(10^{-9} \mathrm{~cm}^{2} \mathrm{~s}^{-1}\right)$, and $\left[\mathrm{H}_{2} \mathrm{SO}_{4}\right]$ is the molecular concentration of $\mathrm{H}_{2} \mathrm{SO}_{4}$. $\mathrm{Nc}$ is the number of $\mathrm{H}_{2} \mathrm{SO}_{4}$ molecules in a critically sized embryo. In stratospheric conditions only molecules of $\mathrm{H}_{2} \mathrm{SO}_{4}$ and water can form stable clusters, and $N c$ is $3(h \leq 27 \mathrm{~km})$ and $10(h>27 \mathrm{~km})$ respectively (Arnold, 1982).

Figure 5 shows the condensation nuclei $(C N)$ profile calculated for the 16 February 1984 GLE event using Eq. (5) and altitude distribution of $\mathrm{H}_{2} \mathrm{SO}_{4}$ for winter conditions (Arnold, 1982). As we can see from the Fig. 5, calculated values of $C N$ are one order greater than experimental ones of $N d$. Note that high and low levels of $C N$ increase coincide with experimental ones. The high level $(h=27 \mathrm{~km})$ corresponds to the altitude of $\mathrm{H}_{2} \mathrm{SO}_{4}$ maximum supersaturation (Arnold, 1982); the low one 


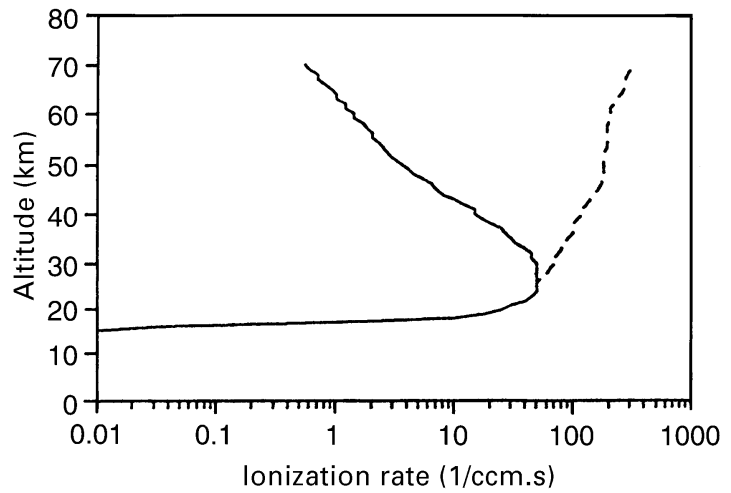

Fig. 6. Ionization rate profiles calculated for GLE event of 16 February 1984 at time of its maximum. Solid curve gives ionization rate profile calculated with geomagnetic cut-off $\mathrm{E}_{1}$ at $150 \mathrm{MeV}$, and dashed curve gives ionization rate profile calculated when $\mathrm{E}_{1}=0$

$(h=16 \mathrm{~km})$ is related to ionization rate decrease (see Fig. 6). But we have to take into account the fact that aerosol particles detected by lidar have radii of more than $0.69 \mu \mathrm{m}$ compared with smaller radii of $C N$ at $0.01 \mu \mathrm{m}$ (Arnold, 1982). These radii differences can be reduced by involving coagulation process in terms of a kinetic equation (Twomey, 1977):

$d N / d t=0.5 \int_{0}^{\infty} \int_{0}^{\infty} K\left(X_{1}, X_{2}\right) n\left(X_{1}\right) n\left(X_{2}\right) d X_{1} d X_{2}$,

where $N$ is the total number of particles, $n\left(X_{1}\right)$ and $n\left(X_{2}\right)$ are the numbers of particles of sizes $X_{1}$ and $X_{2}$ respectively, and $K\left(X_{1}, X_{2}\right)$ is the coagulation coefficient. In our case we have $C N$ where radius is $0.01 \mu \mathrm{m}$ and aerosol particles where radius is more than $0.69 \mu \mathrm{m}$. So, Eq. (6) may be rewritten as:

$d N / d t=-0.5 K_{1} n^{2}-K_{2} n N b$,

where $n$ is the number of calculated $C N$ of radius $0.01 \mu \mathrm{m}$, and $\mathrm{Nb}$ is the number of measured background aerosol particles of radius more than $0.69 \mu \mathrm{m}$. The coefficient $K_{1}$ $\left(24 \cdot 10^{-10} \mathrm{~cm}^{3} \mathrm{~s}^{-1}\right)$ accounts for self-coagulation of $C N$, and the coefficient $K_{2}\left(78000 \cdot 10^{-10} \mathrm{~cm}^{3} \mathrm{~s}^{-1}\right)$ accounts for coagulation of $C N$ with large aerosol particles with radii exceeding $0.69 \mu \mathrm{m}$ (Twomey, 1977).

Using Eq. (7) and assuming $K_{1}$ and $K_{2}$ to be constant we have calculated the time necessary for $C N$ to transform from tiny particles whose radius is $0.01 \mu \mathrm{m}$ to large aerosols with radii exceeding $0.69 \mu \mathrm{m}$ through coagulation process. For $25 \mathrm{~km}$ altitude it takes about 1.5 days and decreases with altitude, so that at $16 \mathrm{~km}$ a time of several hours is needed. In comparison the time scale for adding an $\mathrm{H}_{2} \mathrm{SO}_{4}$ molecule to a $C N$ is approximately $3 \mathrm{~h}$ (Arnold, 1982). Note these values are very variable depending on $\mathrm{H}_{2} \mathrm{SO}_{4}$ concentration and aerosol particle distribution.

We have to consider that some additional physical processes can strongly influence the aerosol formation mechanism for example: additional $\mathrm{H}_{2} \mathrm{SO}_{4}$ sources, such as hydroxyl radicals $(\mathrm{OH})$ that seem to appear during GLE events (Krieger and Arnold, 1994); decrease of stratospheric temperatures during SPE events (Kodama et al., 1992); sudden stratospheric warming and subsequent sudden cooling that can increase level of $\mathrm{H}_{2} \mathrm{SO}_{4}$ supersaturation etc. According to the model suggested the observed $R(H)$ enhancement was caused by sulfate aerosol particles with radii $r \geq 0.69 \mu \mathrm{m}$. It is known that liquid sulfate aerosols of such sizes can exist in the stratosphere under temperatures which are lower than type-1 PSC creation temperatures (Dye et al., 1992; Larsen, 1994). On the other hand, one can not exclude the type-1 PSC creation because the temperatures are quite low enough for it to occur (see Fig. 4). In this case the sulfate aerosol particles may be condensation nuclei for type-1 PSCs (Tolbert, 1994). Some other physical mechanisms are also possible. For example, according to Tinsley et al. (1989) and Tinsley and Heelis (1993), ionization charges supercooled droplets in the stratosphere causing droplet growth and nucleation process intensification.

Results of the work show that GLE events seem to be able to trigger aerosol creation at high latitudes. Some experimental facts confirm the probable connection between solar proton events and aerosol concentration increase (Hofman and Rosen, 1983; Pudovkin and Babushkina, 1992; Roldugin and Vashenyuk, 1994). In Shumilov et al. $(1992 ; 1995)$ reported an ozone total content decrease during a GLE event and Tinsley et al. (1993) have demonstrated the connection between the vorticity atmospheric index (VAI) and galactic cosmic ray variations which also indirectly confirm the reality of such connections.

\section{Conclusions}

1. A considerable increase of aerosol concentration up to $50 \%$ at $20 \mathrm{~km}$ level after GLE event on 16 February 1984 was documented by lidar measurements at Verhnetulomski observatory.

2. The profile of $C N$ calculated using ion nucleation mechanism is presented. By modelling the growth of $C N$ through coagulation process it is shown that theoretical and experimental aerosol altitude profiles coincide at some altitude ranges.

3. The results show the very important additional consequence that the transparency of the atmosphere can be greatly decreased after GLE events.

Acknowledgements. The authors wish to thank E. Friis-Christiansen for supplying the meteorological data. The work was supported by the Russian Foundation of Fundamental Investigations under grant 93-05-12181, INTAS Association under grant 21247/93 and the Norwegian Research Council.

Topical Editor F. Vial thanks a referee for his help in evaluating this paper.

\section{References}

Arnold, F., Ion nucleation - a potential source for stratospheric aerosols, Nature, 299, 134-137, 1982.

Bajdalov, S. I., The employment of lasers in studying the upper atmosphere (in Russian), Ionos. Issled., 26, 5-19, 1978. 
DeLuisi, J. J., B. G. Schuster, and R. K. Sato, Separation of dust and molecular scattering contributions to the lidar observation: a method, Appl. Opt., 14, 1917-1923, 1975.

Dye, J. E., D. Baumgardner, B. W. Gandrud, S. R. Kawa, K. K. Kelly, M. Loewenstein, G. V. Ferry, and B. L. Gary, Particle size distributions in Arctic polar stratospheric clouds, growth and freezing of sulfuric acid droplets, and implications for cloud formation, J. Geophys. Res., 97, 8015-8034, 1992.

Hargreaves, J. K., H. Ranta, A. Ranta, E. Turunen, and T. Turunen, Observations of the polar cap absorption event of February 1984 by the EISCAT incoherent scatter radar, Planet. Space Sci., 35, 947-958, 1987.

Hofman, D. J., and J. M. Rosen, Condensation nuclei events at $30 \mathrm{~km}$ and possible influences of solar cosmic rays, Nature, 302, 511-514, 1983.

Hofman, D. J., and S. Solomon, Ozone destruction through heterogeneous chemistry following the eruption of E1 Chichon, $J$. Geophys. Res., 94, 5029-5041, 1989.

Hofman, D. J., J. M. Rosen, R. Reiter, and H. Jager, Lidar- and balloon-borne particle counter comparisons following recent volcanic eruptions, J. Geophys. Res., 88, 3777-3782, 1983.

Jackman, C. H., J. E. Frederick, and R. S. Stolarski, Production of odd nitrogen in the stratosphere and mesosphere: an intercomparison of source strengths, J. Geophys. Res., 85, 7495-7505, 1980.

Kent, G. S., and R. W. H. Wright, A review of laser radar measurements of atmospheric properties, J. Atmos. Terr. Phys., 32, 917-943, 1970.

Kodama, M., T. Kohno, and H. Kanzawa, Stratospheric sudden cooling after solar proton event over Syowa station, Antarctica, J. Geomagn. Geoelectr., 44, 361-366, 1992.

Krieger, A., and F. Arnold, First composition measurements of stratospheric negative ions and inferred gaseous sulfuric acid in the winter Arctic vortex: implications for aerosols and hydroxyl radical formation, Geophys. Res. Lett., 21, 1259-1262, 1994.

Langner, J., H. Rodhe, P. J. Crutzen, and P. Zimmermenn, Anthropogenic influence on the distribution of tropospheric sulphate aerosol, Nature, 359, 712-715, 1992.
Larsen, N., The impact of freezing of sulfate aerosols on the formation of polar stratospheric clouds, Geophys. Res. Lett., 21 , 425-428, 1994.

Nagatani, R. M., A. J. Miller, M. E. Gelman, and P. A. Newman, A comparison of Arctic lower stratospheric winter temperatures for 1988-89 with temperatures since 1964, Geophys. Res. Lett., 17, 333-336, 1990.

Pudovkin, M. I., and S. V. Babushkina, Influence of solar flares and disturbances of the interplanetary medium on the atmospheric circulation, J. Atmos. Terr. Phys., 54, 841-846, 1992.

Roldugin, V. K., and E. V. Vashenyuk, Atmospheric transparency variations under the influence of the solar cosmic rays (in Russian), Geomagn. Aeron., 34, 155-158, 1994

Russel, P. B., T. J. Swissler, and M. P. McCormick, Methodology for error analysis and simulation of lidar aerosol measurements, Appl. Opt., 18, 3783-3797, 1979.

Shumilov, O. I., K. Henriksen, O. M. Raspopov, and E. A. Kasatkina, Arctic ozone abundance and solar proton events, Geophys. Res. Lett., 19, 1647-1650, 1992.

Shumilov, O. I., E. A. Kasatkina, K. Henriksen, and O. M. Raspopov, Ozone "miniholes" initiated by energetic solar protons, J. Atmos. Terr. Phys., 57, 665-671, 1995.

Tinsley, B. A., and R. A. Heelis, Correlations of atmospheric dynamics with solar activity evidence for a connection via the solar wind, atmospheric electricity, and cloud microphysics, J. Geophys. Res., 98, 10375-10384, 1993.

Tinsley, B. A., G. M. Brown, and P. H. Scherrer, Solar variability influences on weather and climate: possible connections through cosmic ray fluxes and storm intensification, J. Geophys. Res., 94 14783-14792, 1989.

Tolbert, M. A., Sulfate aerosols and polar stratospheric cloud formation, Science, 264, 527-528, 1994.

Tolbert, M. A., M. J. Rossi, and D. M. Golden, Heterogeneous interactions of chlorine, nitrate, hydrogen chloride, and nitric acid with sulphuric acid surfaces at stratospheric temperatures, Geophys. Res. Lett., 15, 847-850, 1988.

Twomey, S., Atmospheric aerosols, Elsevier Scientific, Amsterdam, 576 pp., 1977. 